\title{
Low-Power Implantable Device for Onset Detection and Subsequent Treatment of Epileptic Seizures: A Review
}

\author{
Muhammad Tariqus Salam ${ }^{1}$, Mohamad Sawan', and Dang Khoa Nguyen ${ }^{2}$ \\ ${ }^{1}$ Polystim Neurotechnologies Laboratory, École Polytechnique de Montréal, Québec \\ ${ }^{2}$ Neurology service, Notre-Dame Hospital (Centre Hospitalier de l'Université de \\ Montréal), Montréal, Québec, H3C 3A7, Canada \\ email: tariqus.salam@polymtl.ca
}

\begin{abstract}
Over the past few years, there has been growing interest in neuro-responsive intracerebral local treatments of seizures, such as focal drug delivery, focal cooling, or electrical stimulation. This mode of treatment requires an effective intracerebral electroencephalographic acquisition system, seizure detector, brain stimulator, and wireless system that consume ultra-low power. This review focuses on alternative brain stimulation treatments for medically intractable epilepsy patients. We mainly discuss clinical studies of long-term responsive stimulation and suggest safer optimized therapeutic options for epilepsy. Finally, we conclude our study with the proposed low-power, implantable fully integrated device that automatically detects low-voltage fast activity ictal onsets and triggers focal treatment to disrupt seizure progression. The detection performance was verified using intracerebral electroencephalographic recordings from two patients with epilepsy. Further experimental validation of this prototype is underway.
\end{abstract}

Keywords: Electroencephalographic (EEG), seizure detector, focal drug delivery, focal cooling, electrical stimulation and implantable device

\section{INTRODUCTION}

Approximately 4 million people in North America and at least 50 million people in the world have epilepsy. Epilepsy is a common chronic neurological disorder characterized by a predisposition to unprovoked recurrent seizures. A seizure is the manifestation of an abnormal, hypersynchronous discharge of a population of cortical neurons [1]. Several causes may disturb the normal pattern of brain activity and trigger an epileptic seizure, such as head injury, brain infection, developmental malformations, brain tumors, cerebrovascular disease, vascular malformations, genetic disorders, and hippocampal sclerosis.

Epileptic seizures may loosely be classified into two categories: focal (partial) and generalized seizures. Focal indicates that the seizures originate primarily within networks limited to one cerebral hemisphere, discretely localized (arrows in Fig. 1a) or 
(a)

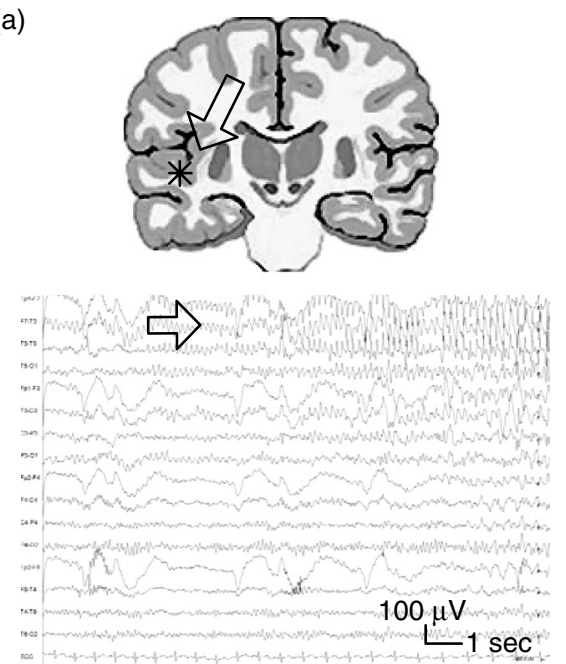

(b)

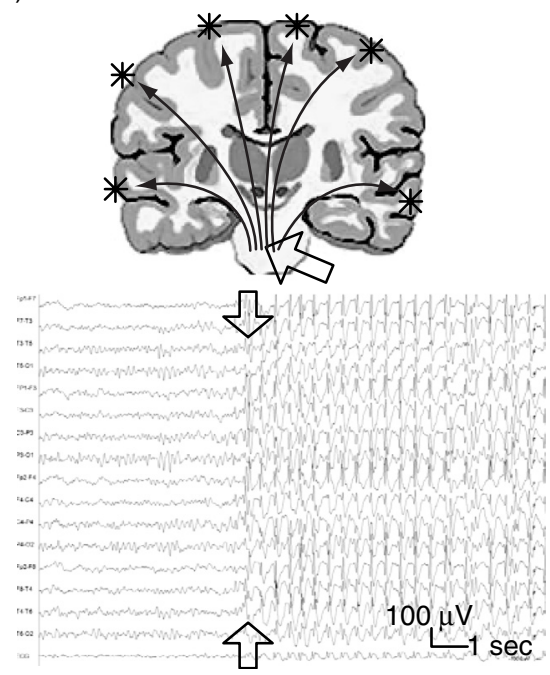

Figure 1. Typical epileptic seizure (a) partial and (b) generalized seizures.

more widely distributed. Focal seizures may secondarily generalize as the epileptic discharge spreads contralaterally.

Primary generalized epileptic seizures originate within, and rapidly engage, bilaterally distributed networks (arrows in Fig. 1b). Figure 1 shows the invasive EEG acquisition, which helps to find seizure type and epileptogenic zones. In this figure, the partial seizure shows the sudden appearance of this typical low-voltage fast activity recorded from the intracerebral contacts positioned over the epileptogenic zone (arrows in Fig. 1a) and the generalized seizure shows the rhythmic pattern activity recorded from many intracerebral contacts over a large area of brain (arrows in Fig. 1b). However, electrographically, many seizure patterns are seen at seizure onset and there is variability in the seizure pattern depending on the localization and etiology of the seizure. The most common seizure onset patterns are low-voltage fast activity, highvoltage fast activity, and the rhythmic spiking [1]. Clinically, these ictal discharges usually lead to behavioral manifestations but may also be clinically silent (i.e., electrical seizures), especially if the ictal discharges remain very focal (without spread), are brief (a few seconds) and occur in non-eloquent cortex.

Antiepileptic drugs are the mainstay of treatment, but many patients have systemic and central nervous system side effects and a third of them are refractory. Because most drug-resistant epileptics suffer from focal epilepsy, these patients may benefit from epilepsy surgery. Several studies have shown that resection of the epileptogenic zone may lead to seizure freedom [2]. Success is dependent on the accurate localization and complete resection of the epileptogenic zone. Unfortunately, not all refractory patients with focal epilepsy benefit from resective surgery; some have an epileptogenic zone overlying eloquent areas (language, primary motor or visual areas) that cannot be 
resected without permanent sequelae, while others have multifocal epilepsy. Therefore, the poor neurological outcome of these cases combined with the lack of efficacy and adverse effects of antiepileptic drugs provide sufficient justification to have an alternative treatment to supplement conventional treatments. Given the therapeutic needs, neurostimulation has been proposed as an alternative treatment.

Over the last quarter of the century, the vast progression in neuro-technology has allowed the development of FDA-approved stimulators for the treatment of neurological disorders, such as multi-channel cochlear implant for hearing loss, deep brain simulation for Parkinson's disease, and vagus nerve simulation (VNS) for epilepsy. VNS provides scheduled stimulation (open-loop) to the left vagus nerve to reduce seizure frequency by a yet unclear mechanism. However, seizure freedom is rare, and only $30-40 \%$ of patients show a significant decrease in seizure frequency [3]. More recently, there has been growing interest in the development of responsive therapeutic devices to abort seizures at their onset. The responsive device (detection and treatment) identifies seizures at their onset and triggers focal treatment to the epileptogenic zone to abort the seizure, whether by electrical stimulation, cooling, or drug release. To do so, an efficient seizure detection algorithm is required for accurate seizure onset detection without false alarms, and which can be implemented in a custom integrated circuit. Already, one such type of device is undergoing clinical trial for refractory partial epilepsy: the cranially implanted Responsive Neurostimulator (RNS) [3].

In this review, we discuss the novel alternative approaches to the treatment of epilepsy and propose a low-power implantable detection and treatment device.

\section{NEUROSTIMULATION TREATMENT OF REFRACTORY EPILEPSY}

Drug-resistant partial epileptics are not considered to be good candidates for epilepsy surgery; thus, alternative treatments are required. Many studies suggest that the new technologies are promising for the treatment of epilepsy. Given the experiences to date, focal brain stimulation therapy has proven to be an effective therapy and has been receiving attention as an alternative therapy for refractory patients. There are two approaches to brain stimulation: open- and closed-loop. In the former, brain stimulation is scheduled, while in the latter the stimulation is triggered upon detection of a seizure [3]. The advantages of the closed-loop stimulation over the open-loop method are (i) lower number of treatments, (ii) lower adverse effects, (iii) high efficiency because of rapid access, and (iv) shorter investigation [4]. However, the disadvantages are the needs to identify the epileptogenic zone, to distinguish ECoG patterns and to stimulate the brain at earlier stage. The details of the closed-loop brain stimulation approach are discussed below.

\subsection{Closed-Loop Responsive Treatment for Epilepsy}

Proof-of-concept experiments conducted in animals and humans with epilepsy have demonstrated that focal electrical, thermal, or pharmacological manipulations of the epileptogenic zone can suppress seizure activity, paving the way for a novel approach to the treatment of epilepsy [3]. Some of these experiments demonstrated the feasibility of responsive treatment [3-5]. The responsive treatment should be able to analyze the 
intracerebral EEG, detect the seizure onset, and deliver focal treatment to inhibit the epileptiform activity. However, several issues remain to be addressed, such as the necessity of a reliable seizure detection system sensitive enough to detect seizures early on but also specific enough to prevent unwarrantedly triggered focal intervention. Algorithms of the seizure detection and types of brain stimulation are described below.

\subsubsection{Seizure Detection}

Several mathematical models have been developed to detect seizures. Intracerebral EEG-based models have shown much better performance than scalp EEG-based models, as lesser artifacts are encountered with the former. Table 1 briefly summarizes different detection algorithms.

These models were developed using desktop computers for off-time data processing. These types of algorithms cannot be employed in a low-power implantable microchip. So far, very few implantable devices have been proposed for seizure detection. A seizure detection algorithm [18] was proposed for an implantable device and realized with a finite impulse response (FIR) band-pass filter and 15 amplifiers with each amplifier implemented with DC gain, input noise, and GBW of $100 \mathrm{~dB},<200 \mu \mathrm{Vrms}$ and $35 \mathrm{kHz}$, respectively.

An epileptic seizure detector in a custom low-power CMOS integrated circuit that could run for 7 to 10 years with a rechargeable battery was designed and implemented by our team $[19,20]$. The details of this detector are discussed in section 3 .

\subsubsection{Focal Electrical Stimulation}

In a pilot trial, Osorio et al. demonstrated the feasibility and short-term safety of automated high-frequency electrical stimulation in blocking seizures using an external prototype comprising an electrocorticography acquisition system, a computer, and a stimulator (Grass S12) [4]. Another external responsive neurostimulator (eRNS) has been shown to terminate electrographic seizures as well [5]. Apart from seizure detection performance, the other concerns are the safety of chronic electrical stimulation and the determination of optimal stimulation parameters. It is generally agreed that limiting the maximum charge density to $30 \mu \mathrm{C} / \mathrm{cm}^{2}$ per phase can avoid tissue damage [21]; however, no cell damage in human cortex has been found for 50 to $60 \mu \mathrm{C} / \mathrm{cm}^{2}$ per phase stimulation [22]. In fact, continuous deep brain stimulation (DBS) featuring, for example, 1-10 V, $90 \mu \mathrm{sec}$ pulse width, 100-165 Hz frequency for the treatment of Parkinson's disease has been shown to be safe and effective as evidenced by US-FDA approval [21]. A 1 minute on and 5 minute off stimulation paradigm was used during an investigational DBS trial for epilepsy [3, 21].

Most of the reported experiments have shown abortion of seizure activity by electrical stimulation, but there is indication that low-frequency stimulation (lower than $10 \mathrm{~Hz}$ delivered to either posterior hypothalamus or thalamus) may induce a seizure $[3,21]$. It may very well be that proper stimulation parameters need to be individualized during the presurgical intracerebral EEG study for maximum efficacy prior to the implantation of a closed-loop responsive stimulation device. Many stimulation parameters have shown promising performance. For example, Osorio et al. used a 
Table 1. Summary of seizure detection algorithms

\begin{tabular}{|c|c|c|c|}
\hline Authors & Method & Description & Application \\
\hline $\begin{array}{l}\text { McSharry } \\
\text { et al. [6] }\end{array}$ & $\begin{array}{l}\text { Multi-modal } \\
\text { probability } \\
\text { evaluation }\end{array}$ & $\begin{array}{l}\text { Time evolution of the } \\
\text { probability density function. }\end{array}$ & SESD \\
\hline Shoeb et al. [7] & $\begin{array}{l}\text { Wavelet and } \\
\text { neural network }\end{array}$ & $\begin{array}{l}\text { Wavelet decomposition } \\
\text { constructs feature vector and } \\
\text { the support-vector machine } \\
\text { classification algorithm } \\
\text { that determine seizure onset. }\end{array}$ & SESD \\
\hline $\begin{array}{l}\text { Suffczynski } \\
\text { et al. [8] }\end{array}$ & $\begin{array}{l}\text { Bistable } \\
\text { mathematical } \\
\text { model }\end{array}$ & $\begin{array}{l}\text { Predict time of seizure occurrences } \\
\text { using a Poisson process and a } \\
\text { random walk process. }\end{array}$ & IEBSD \\
\hline Moxon et al. [9] & Correlation & $\begin{array}{l}\text { Similarity between a reference } \\
\text { and dynamically recorded signal. }\end{array}$ & IEBSD \\
\hline $\begin{array}{l}\text { Iasemidis et al. } \\
\quad[10]\end{array}$ & $\begin{array}{l}\text { Nonlinear, } \\
\text { Lyapunov } \\
\text { exponent }\end{array}$ & $\begin{array}{l}\text { Convergence and divergence of } \\
\text { short-term maximum Lyapunov } \\
\text { exponents (STLmax) among } \\
\text { critical electrode sites. }\end{array}$ & IEBSD \\
\hline $\begin{array}{l}\text { Iasemidis et al. } \\
\text { [11] }\end{array}$ & Phase changes & $\begin{array}{l}\text { Measure average angular } \\
\text { frequency and optimize } \\
\text { the techniques. }\end{array}$ & IEBSD \\
\hline $\begin{array}{l}\text { Mormann et al. } \\
\text { [12] }\end{array}$ & Coherence & $\begin{array}{l}\text { Statistical measurement of phase } \\
\text { synchronization of signals. }\end{array}$ & IEBSD \\
\hline $\begin{array}{l}\text { Tetzlaff } \\
\text { et al. [13] }\end{array}$ & $\begin{array}{l}\text { Features } \\
\text { extraction }\end{array}$ & $\begin{array}{l}\text { Automated technique for the } \\
\text { detection of a preseizure state. }\end{array}$ & IEBSD \\
\hline Arnhold et al. [14] & $\begin{array}{l}\text { Signals } \\
\text { synchronization }\end{array}$ & $\begin{array}{l}\text { Detect weak interdependences } \\
\text { of signal. }\end{array}$ & IEBSD \\
\hline Nagaraj et al. [15] & Synchronization & $\begin{array}{l}\text { Cross-correlationship used to } \\
\text { find dissimilarity of signals } \\
\text { between interictal periods } \\
\text { and epileptic seizures. }\end{array}$ & IEBSD \\
\hline Sukhi et al. [16] & Bayes' theorem & $\begin{array}{l}\text { Spectral feature extraction, Bayes' } \\
\text { theorem and spatiotemporal } \\
\text { analysis. }\end{array}$ & IEBSD \\
\hline Yadav et al. [17] & $\begin{array}{l}\text { Evolution-based } \\
\text { data adaptive } \\
\text { classifier }\end{array}$ & $\begin{array}{l}\text { Temporal evolution-based nonlinear } \\
\text { data adaptive classifier utilizing } \\
\text { linear and nonlinear features and } \\
\text { mimics human experts for } \\
\text { classification. }\end{array}$ & IEBSD \\
\hline
\end{tabular}

SESD: Scalp EEG recording and software in desktop computer.

IEBSD: Intracranial electrodes, band pass filter and software in desktop computer. 
biphasic, charge-balanced square pulse (100 $\mu \mathrm{sec} /$ phase width, $100-500 \mathrm{~Hz}$ frequency) for $1 \mathrm{sec}$ [4]. If the stimulation failed to terminate the seizure, the next seizure detection begins within 1 minute. A maximum of five stimulations are allowed to terminate a seizure [4]. In another example, brief bursts of $50 \mathrm{~Hz}$ biphasic stimulation for $0.3-2 \mathrm{sec}$ delivered via subdural electrodes were used [21]. The RNS also uses biphasic, chargebalanced electric pulses $(1-200 \mathrm{~Hz}$ frequency, 0.5 to $12 \mathrm{~mA}$ current, 40 to $1000 \mu \mathrm{s}$ pulse width, burst duration $\leq 5 \mathrm{sec}$, and 1 to 5 bursts) [3, 22]. Our research team has so far proposed some implantable electrical stimulators for different applications, with a wide range of selectable stimulation parameters that provide a flexible framework for intervention via a low-power stimulator for the treatment of epilepsy [23, 25].

\subsubsection{Focal Drug Delivery}

The currently available orally administrated antiepileptic drugs are associated with variable systemic side effects (adverse effects in liver, bone marrow, and central nervous system). Focal drug delivery directly onto the epileptogenic zone could enhance the efficacy of drugs while limiting side effects to a minimum. Experiments in the past showed that focal diazepam infusion using a programmable pump in response to automated seizure detection could suppress the seizure [26, 27]. Other drugs such as DP-valproic acid [28], gabapentin [29], and adenosine [30] have been used but with less success. Much work lies ahead prior to clinical application in optimal drug selection, the delivery system, automated seizure detection, the refilling procedure, etc. To date, technological barriers limit the exploration of direct drug delivery for the long-term treatment of focal epilepsy.

\subsubsection{Focal Cooling with Thermoelectric Devices}

Similar to focal drug delivery, focal cooling triggered by the detection of a seizure may represent an efficient approach to abort seizures on demand without the risk of systemic side effects. Cooling can reduce end-plate potentials, alter the neuronal action potential, inhibit the Na-K ATPase [31], reduce the release of presynaptic transmitters, and decrease neuronal conduction [32,33]. Recent studies using miniaturized Peltier devices constitute proof-of-concept that focal cooling can be effective in suppressing seizure activity [34-36]. However, issues pertaining to high-power consumption and heat dissipation (risk of thermal damage to adjacent tissues) still need to be addressed.

\subsection{Implantable Devices for the Treatment of Epilepsy}

Few implantable devices for the treatment of epilepsy have been commercialized. The only FDA-approved implantable device for the treatment of epilepsy is the vagal nerve stimulator (VNS). The VNS is an open-loop (non-responsive) system comprising an implantable stimuli generator, a lead incorporating a bipolar electrode, and an external pulse programming system for the stimuli generator (see table 2).

The stimuli generator of the VNS is implanted underneath the left clavicle under general anesthesia. The lead is tunnelled to the neck, and the two helical bipolar stimulating electrodes are placed around the left vagus nerve. After the operation, the VNS can be turned on by a computer and programming wand. The most common 
Table 2. Comparison of two commercially available neurostimulators for the treatment of epilepsy

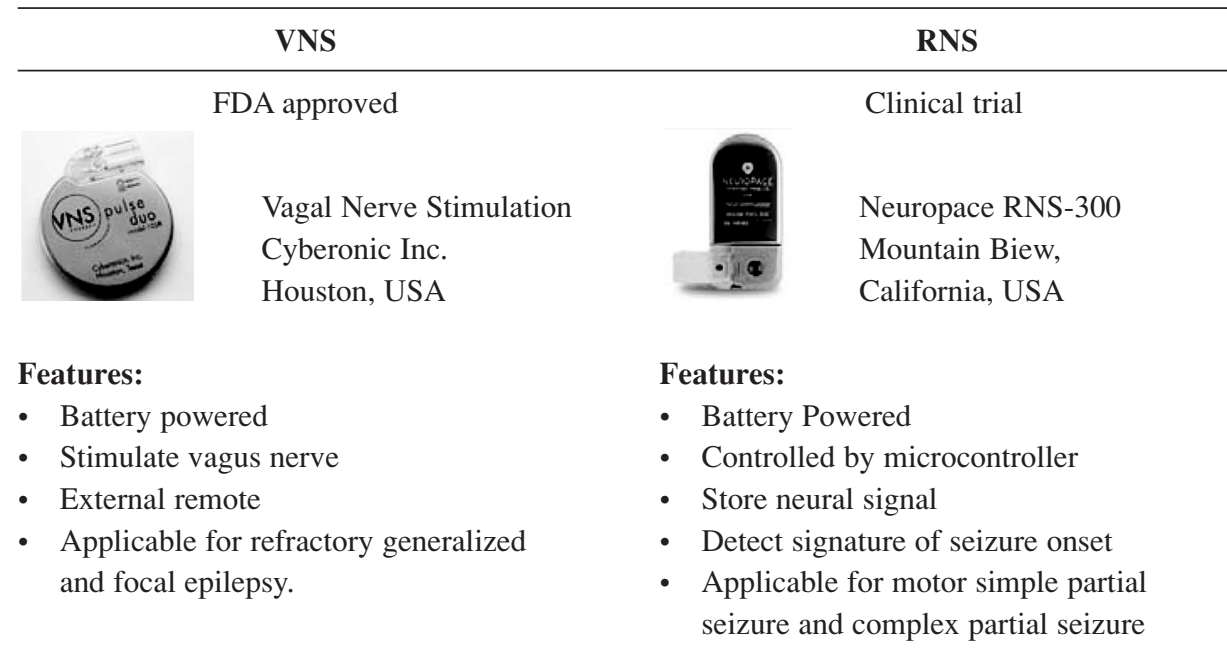

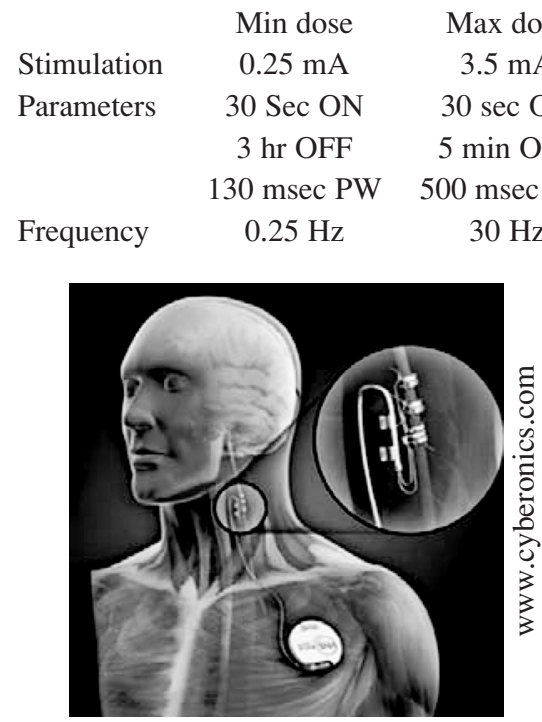

Source: $[3,37-38]$

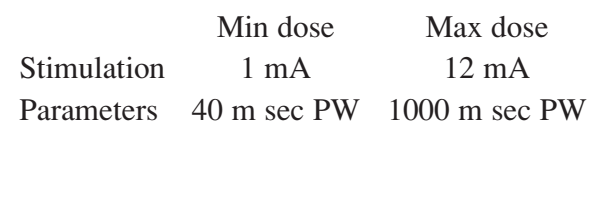

Frequency $\quad 1 \mathrm{~Hz} \quad 333 \mathrm{~Hz}$

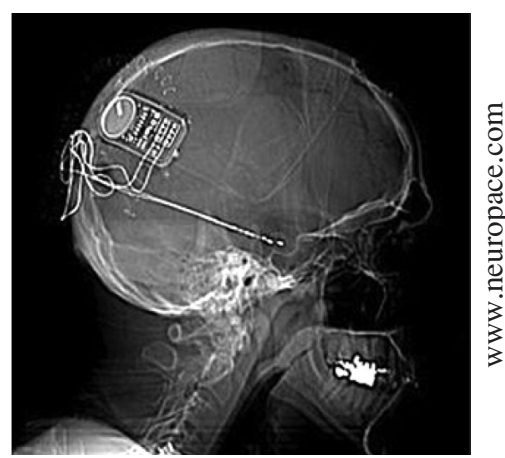

Source: $[3,22,39]$

settings for the stimulator are a frequency of $20-30 \mathrm{~Hz}$, a pulse width of $250-500 \mu \mathrm{s}$, time on of $30 \mathrm{~s}$, and time off of 3-5 min. The advantages of VNS include (a) no need of the exact delineation of the epileptogenic zone, (b) no craniotomy, (c) a lower rate of surgical complications, and (d) mild and infrequent side effects due to stimulation 
(hoarseness, cough). The main disadvantage is that, while $30-40 \%$ of cases show a reduction in seizure frequency of more than $50 \%$, only about $3 \%$ attain seizure freedom (long-term effect on seizure frequency) [37-38].

DBS has also been tried for treating epilepsy by providing scheduled stimulation (open-loop) via depth electrodes to such targets as the hippocampus, thalamus, cerebellum, caudate nucleus and centromedian thalamus, anterior thalamic nucleus, and neocortical seizure foci $[39,40]$. However, the mechanisms of DBS for stimulation of the brain in the treatment of epilepsy is yet unknown, and unfortunately, the results so far have been contradictory [3, 40].

More recently, the cranially implanted Responsive Neurostimulator (RNS, closedloop) system has been proposed for the treatment of refractory epilepsy [3]. It consists of a programmable, battery-powered microprocessor-controlled device (the RNS neurostimulator) implanted in the cranium, which delivers a short train of electrical pulses to the epileptogenic zone through intracranial electrodes (depth and cortical strip leads) when seizure onset is detected. An external programmer, laptop computer, wand, and telemetry interface enable communication with the implanted RNS neurostimulator, allowing the physician to modify the parameters of detection and stimulation of RNS through wireless communication. This investigational responsive device is still under clinical trial $[22,39]$. Table 2 compares the RNS features with those of the VNS.

\section{THE PROPOSED IMPLANTABLE DEVICE FOR EPILEPSY TREATMENT}

The recent surging interest and growing clinical needs have motivated us to propose a fully integrated device which includes a low-power detector and stimulator as well as a drug-delivery bio-MEMS-based module. This device is intended to perform efficient seizure detection and subsequent treatment. The proposed closed-loop automated system is designed for patients with partial (focal) seizure. The proposed device contains a patient-specific (tunable) seizure onset detector and controllable stimuli generator. The overall system will be triggered by the electrical stimulation of a targeted brain area in response to a low-voltage fast activity seizure pattern. Figure 2 shows an intracerebral recording of a typical low-voltage fast activity as seen at seizure onset over the epileptogenic area, with subsequent evolution in frequency and amplitude as the seizure progresses and propagates to other electrode contacts. The proposed system detects the seizure onset and stimulates neural tissues to terminate the seizure progression and propagation.

Figure 3a illustrates the implant configuration while Figs. $3 \mathrm{~b}$ and $3 \mathrm{c}$ show the block diagram of the proposed implantable device and the flowchart of the closed-loop automated system, respectively. Wireless transmission to an external device allows the clinician to review intracerebral recordings, and to modify the parameters of seizure detection and electrical stimulation as required. The flowchart of the closed-loop system shows that electrical stimulation therapy is activated in response to seizure detection.

Our research group has proposed several low-power implantable preamplifiers for neural signal amplification [41-46] and more recently, low-power implantable 


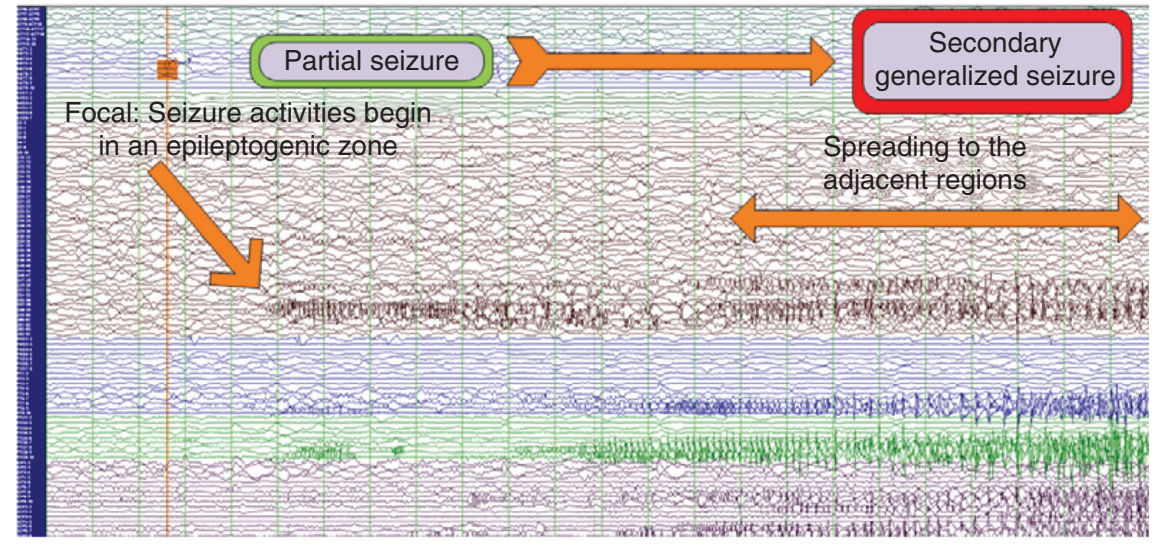

Figure 2. Intracerebral EFG recordings of a patient with refractory focal epilepsy [source: Centre Hospitalier de 1'Université de Montréal].

(a)

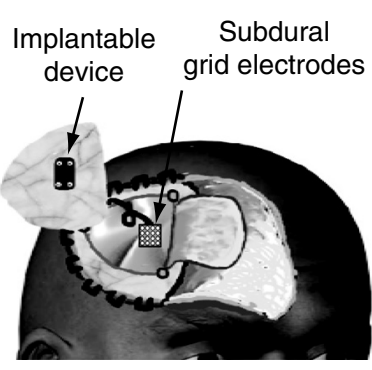

(b)

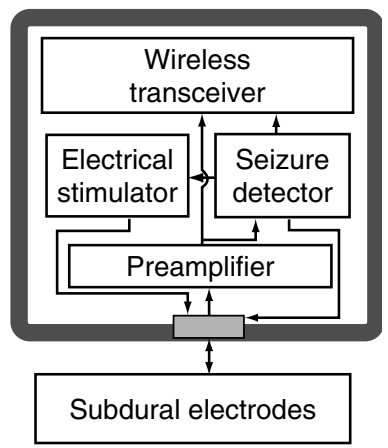

(c)

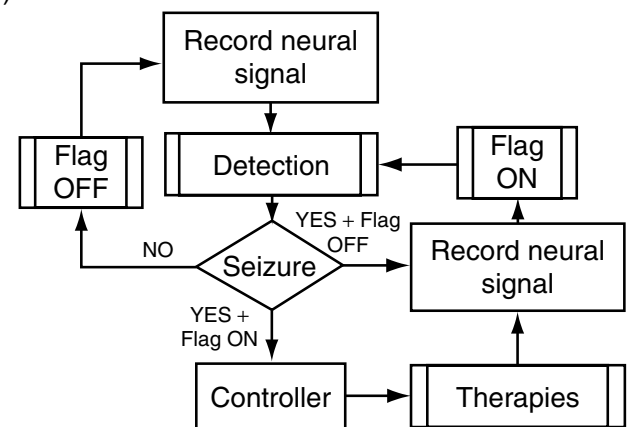

Figure 3. (a) Illustration of the implant configuration, (b) block diagram of the implantable device, and (c) flowchart of the closed-loop automated system. 
integrated devices for automatic seizure detection $[19,20]$. The experimental validation of our seizure detector prototype is under-way. The proposed implantable seizure onset detector (SOD) provides continuous long-term monitoring of intracerebral EEG. The sensitivity of the SOD is enhanced, and several decision boundaries are introduced to reduce the number of false alarms based on the patient's specific seizure pattern. Signal analysis of this SOD demonstrates that through early modulation and proper rectification of the intracerebral signal, the seizure onset information can be efficiently extracted. Therefore, the detected hyperexcitation of the intracerebral signal is accurately analyzed over a certain time frame. The duration of the detected hyperexcitation then indicates an upcoming seizure event. Furthermore, this SOD determines the highfrequency patterns and the progressive amplitude increase of the seizure signal. Because the ictal patterns vary from patient to patient according to the underlying substrate, the type of intracerebral electrodes used, and their locations with respect to the epileptogenic zone, the seizure detection algorithm requires several adjustable parameters for optimal sensitivity and specificity.

As some patients with refractory epilepsy also present numerous asymptomatic brief electrical seizures (in addition to the less frequent electroclinical seizures), the physician may decide to adjust the seizure detection parameters to trigger or withhold focal stimulation at those asymptomatic discharges. For some patients, one could elect not to trigger stimulation (or any other type of focal treatment) at these frequent, brief and silent events to spare battery life.

Figure 4 shows the intracerebral EEG recording of a brief electrical seizure followed by an electroclinical seizure. The recording is analyzed with Matlab software, and a seizure detection algorithm for partial-onset seizures is employed. In this algorithm, the input signal is modulated into high frequency $\left(F_{\mathrm{S}}=1 / T_{\mathrm{s}}\right)$ so that instrumentation lowfrequency noise does not affect the signal. The discrete signal confined to a time frame $\left(T_{f}\right)$ passes through $\mathrm{N}$ number of voltage level detectors (VLD) to detect the specific features of a progressive hyperexcited signal. The threshold voltages of VLD and $T_{f}$ are tuned to the specific seizure onset frequency of a patient to minimize false alarms.

Figure $4 \mathrm{a}$ demonstrates the seizure detection algorithm on a signal confined to $T_{f}=$ $2 \mathrm{sec}$. The normal, electrical seizure and electroclinical seizure patterns are modulated (Figs. 4a1-4a14), and each of these modulated signals pass through four VLDs (threshold voltages are shown in Fig. 4b1). The high number of detections in Fig. $4 \mathrm{~b} 1-4 \mathrm{~b} 14$ reflects the high frequency of the signal and the similar numbers of detection represent progressive increases in the signal as seen during a seizure. Figures $4 \mathrm{c} 1-4 \mathrm{c} 14$ show the frequency analysis of these signal patterns. Hence, the algorithm is able to determine the high frequency as well as the progressive increase in the amplitude (Fig. 4b12-4b13) of the seizure signal. To further minimize false alarms, an adjustable time frame (two seconds in this example) of high-frequency electrical seizure is set (Fig. 4c6-4c7). The detection performance was recently verified using intracerebral EEG recordings from different patients with refractory epilepsy. A 30-year-old patient with intractable epilepsy underwent left craniotomy for invasive EEG evaluation, and the detection performance of a low power $(6.71 \mu \mathrm{W}) \mathrm{CMOS}$ integrated low-voltage fast activity seizure onset detector was verified using this recorded EEG signal [20]. 
(a)
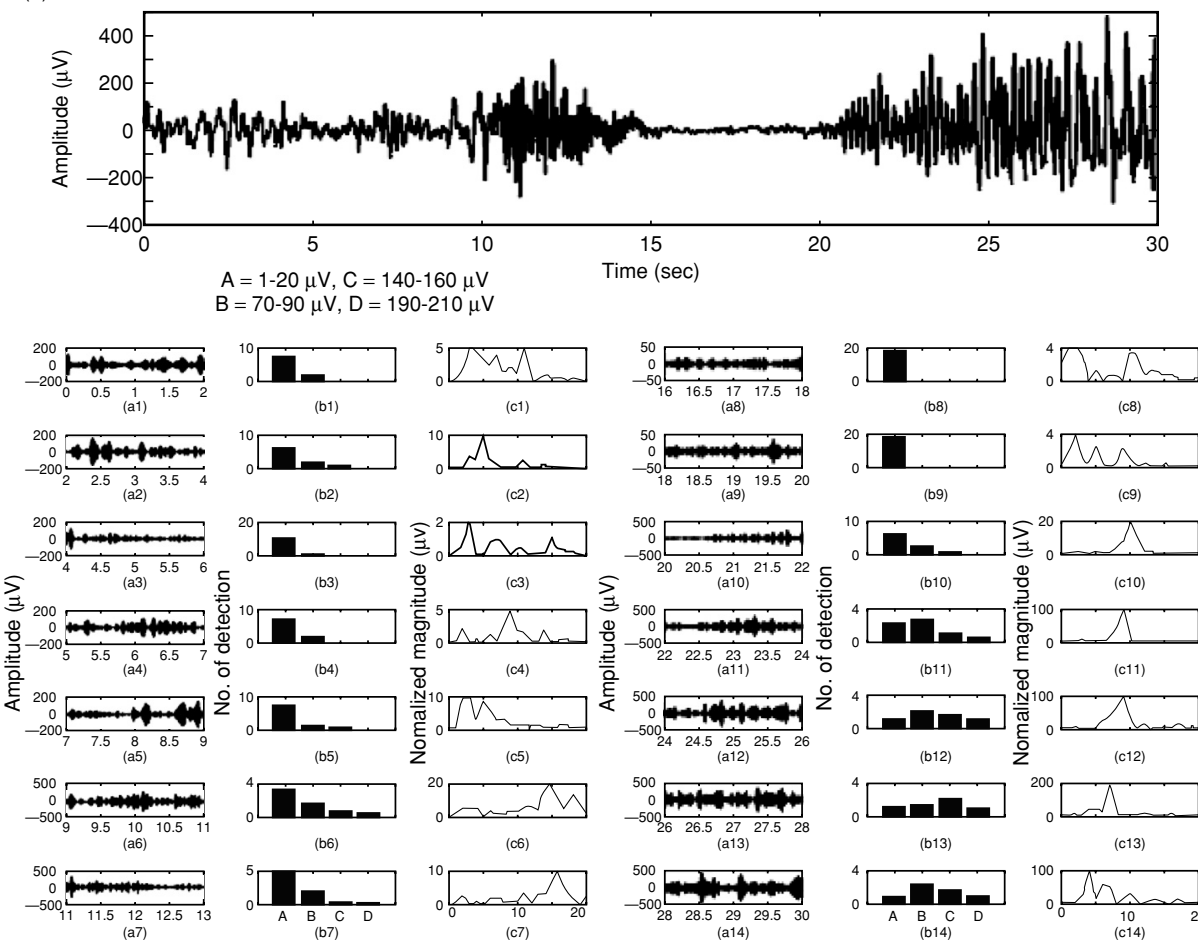

(c2)
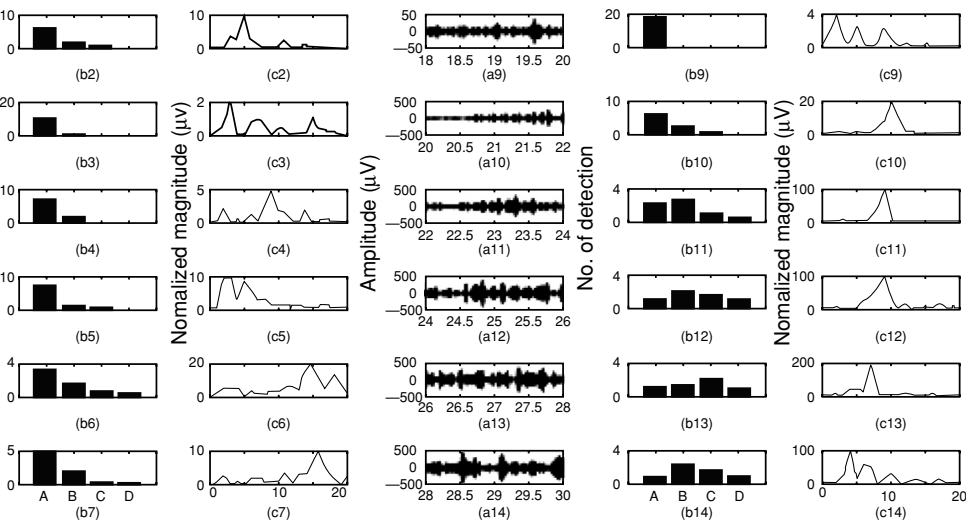

Figure 4. Detection of an epileptic seizure onset: (a) Invasive EFG recording of a patient with refractory focal epilepsy, (a1)-(a14) are modulated signals for every $2 \mathrm{sec}$ of (a), (b1)-(b14) are the output of the voltage level detector, and (c1)-(c14) show the frequency analysis of the signals for every $2 \mathrm{sec}$ of (a).

Another detector $(6.72 \mu \mathrm{W})$ was verified using intracerebral electroencephalographic recordings from a 27-year-old patient with intractable epilepsy and the detector accurately identified seizure onsets [19].

The seizure detector enables the electrical stimulator to deliver a burst of electrical pulses via intracranial electrodes (Fig. 3a). The dimensions of these intracranial electrodes could be relatively standard (e.g., 2-4 mm in diameter), and the interelectrode spacing of $10 \mathrm{~mm}$ [1] or custom made (Fig. 5a) depending on the size and location of the epileptogenic zone as delineated from an in-patient invasive monitoring study.

Effective individualized stimulation parameters could also have been identified during the in-hospital invasive monitoring study. With respect to the safety limits of chronic electrical stimulation, the proposed system can provide a high-frequency (100 to $500 \mathrm{~Hz}$ ) biphasic and charge-balanced square wave (Fig. 5b) up to a maximum of 
(a)

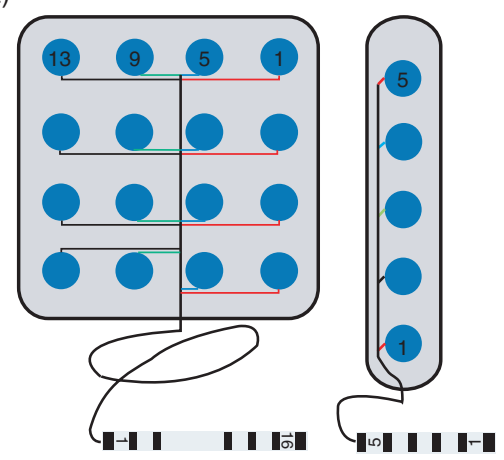

(b)

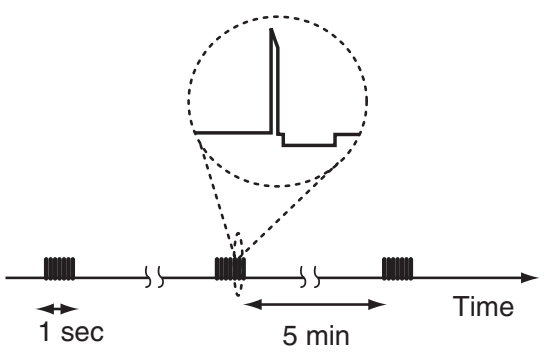

Figure 5. (a) The custom-made subdural electrodes and (b) the biphasic chargebalanced electrical stimulation.

five stimulations for a seizure. If desired, the clinician could always modify the stimulation parameters through wireless communication. For example, the low-power wireless transceiver RFM TRC-104 (RF Monolithics, Inc.) is known to be reliable, safe, and flexible for data transmission. Overall, the total estimated power consumption of the proposed system is less than $13 \mathrm{~mW}$. Therefore, the whole device could theoretically run on a button lithium ion battery for 2 to 3 years. Replacing the battery would not require a major surgical procedure.

\section{CONCLUSION}

Drug-resistant patients with epilepsy may be candidates for non-pharmacological therapies, such as implantable medical devices. So far, three devices are commercially available for the treatment of epilepsy, and a number of strategies hold promise for improving their effectiveness. There are advantages and drawbacks of these available implantable devices, specifically in seizure onset detection and neurostimulation of cortical epileptogenic focus to stop the progression of seizures. Closed-loop implantable responsive systems for the focal treatment of refractory partial epilepsy are promising therapeutic avenues that show potential for effective treatment with minimal side effects. A fully integrated tunable implantable system responsive to ictal low-voltage fast activity patterns is being developed with a wireless fully programmable neurostimulator integrated to ensure precise interaction with the epileptogenic focus. Novel approaches in direct electrical stimulation, drug delivery, thermal cooling, and new subdural electrode design should offer a chance for long-term seizure remission or even cure.

\section{ACKNOWLEDGMENTS}

The authors are grateful for the support from the Natural Sciences and Engineering Research Council (NSERC), the Canada Research Chair in Smart Medical Devices, the Microsystems Strategic Alliance of Québec (ReSMiQ), and the EEG technicians at Notre-Dame Hospital of Montréal. 


\section{REFERENCES}

[1] Nguyen, D. K. and Spencer, S. S., Invasive EEG in presurgical evaluation of epilepsy, in Chapter 53 of The Treatment of Epilepsy, 2nd edn., Wiley-Blackwell, 2004, 633-658.

[2] Jerome, E., J., Overview of surgical treatment for epilepsy, in Chapter 60 of The Treatment of Epilepsy, 3rd edn., Wiley-Blackwell, 2009, 743-756.

[3] Schachter, S. C., Guttag, J., Schiff, S. J., Schomer, D. L. and Summit Contributors, Advances in the Application of Technology to Epilepsy: The CIMIT/NIO Epilepsy Innovation Summit, Epilepsy \& Behavior, 2009, 16, 3-46.

[4] Osorio, I., Frei, M. G., Sunderam, S., Giftakis, J., Bhavaraju, N. C., Schaffner, S. F. and Wilkinson, S. B., Automated seizure abatement in humans using electrical stimulation, Annals of Neurology, 57(2), 2005, 258-68.

[5] Kossoff, E. H., Ritzl, E. K., Politsky, J. M., Murro, A. M., Smith, J. R., Duckrow, R. B., Spencer, D. D. and Bergey, G. K., Effect of an external responsive neurostimulator on seizures and electrographic discharges during subdural electrode monitoring, Epilepsia, 45, 2004, 1560-1567.

[6] McSharry, P. E., He, T., Smith, L. A. and Tarassenko, L., Linear and non-linear methods for automatic seizure detection in scalp electro-encephalogram recordings, Medical \& biological engineering \& computing, 40(4), 2002, 447-461.

[7] Shoeb, A., Edwards, H., Connolly, J., Bourgeois, B., Treves, T. and Guttag, J., Patient-Specific Seizure Onset Detection, Engineering in Medicine and Biology Society, 5(4), 2004, 483-498.

[8] Suffczynski, P., Da Silva, F. H. L., Parra, J., Velis, D. N., Bouwman, B. M., van Rijn, C. M., van Hese, P., Boon, P., Khosravani, H., Derchansky, M., Carlen, P. and Kalitzin, S., Dynamics of epileptic phenomena determined from statistics of ictal transitions, IEEE Transactions on Biomedical Engineering, 53(3), 2006, 524-532.

[9] Moxon, K., Kuzmick, V., Lafferty, J., Serfass, A., Szperka, D., Zale, B., Johnson, J. and Nagvajara, P., Real-time seizure detection system using multiple single-neuron recordings, Conference Proceedings of the 23rd Annual International Conference of the IEEE Engineering in Medicine and Biology Society, Turkey, 2001, 934-937.

[10] Iasemidis, L. D., Deng-Shan, S., Chaovalitwongse, W., Sackellares, J. C., Pardalos, P. M., Principe, J. C., Carney, P. R., Prasad, A., Veeramani, B. and Tsakalis, K., Adaptive epileptic seizure prediction system, IEEE Transactions on Biomedical Engineering, 50, pp., 2003, 616-627.

[11] Iasemidis, L. D., Pardalos, P. M., Shiau, D.S., Chaowolitwongse, W., Narayanan, K., Kumar, S., Carney, P. R., and Sackellares, J.C., Prediction of human epileptic seizures based on optimization and phase changes of brain electrical activity, J. Optimization Methods and Software, 18, 2003, 81-104.

[12] Mormann, F., Lehnertz, K., David, P., and Elger, C. E., Mean phase coherence as a measure for phase synchronization and its application to the EEG of epilepsy patients, Elsevier Science, 144, 2000, 358-369.

[13] Tetzlaff, R., Niederhofer, C. and Fischer P., Automated detection of a preseizure state: nonlinear EEG analysis in epilepsy by cellular nonlinear networks and Volterra systems, International Journal of Circuit Theory and Applications, 34, 2006, 89-108.

[14] Arnhold, J., Grassberger, P., Lehnertz, K. and Elger, C. E. A robust method for detecting interdependences: application to intracranially recorded EEG, Physica D: Nonlinear Phenomena, 134, 1999, 419-430.

[15] Nagaraj, S., Shah, A., Shah, P., Szeto, V. and Bergen, M. T., Ambulatory preseizure detection device, IEEE 32nd Annual Northeast Bioengineering Conference, Easton, PA, USA, 2006, 41-42.

[16] Sukhi, G. and Jean, G., An automatic warning system for epileptic seizures recorded on intracerebral EEGs, Clinical neurophysiology, 116, 2005, 2460-2472.

[17] Yadav, R., Agarwal, R. and Swamy, M. N. S., A novel dual-stage classifier for automatic detection of epileptic seizures, Engineering in Medicine and Biology Society, 30th Annual International Conference of the IEEE, 2008, 911-914. 
[18] Bhavaraju, N. C., Frei, M. G. and Osorio, I., Analog seizure detection and performance evaluation, IEEE transactions on bio-medical engineering, 53, 2006, 238-245.

[19] Salam, M. T., Sawan, M., Hamoui, A. and Nguyen D. K., Low-power CMOS-based epileptic seizure onset detector," IEEE-NEWCAS, French, 2009.

[20] Salam, M. T., Sawan, M., Nguyen, D. K. and Hamoui, A. A. "Epileptic Low-Voltage Fast-Activity Seizure-Onset Detector," IEEE-BIOCAS: special session, China 2009.

[21] Theodore, W. H. and Fisher, R. S., "Brain stimulation for epilepsy," Neurology, 3(8), 2004, 111-118.

[22] Skarpaas, T. L. and Morrell, M. J., Intracranial stimulation therapy for epilepsy, Neurotherapeutics, 6(2), 2009, 238-43.

[23] Coulombe, J., Sawan, M. and Gervais, J. F., A Highly Flexible System for Microstimulation of the Visual Cortex: Design and Implementation, IEEE Trans. Biom. Circuits \& Systems, 1, 2007, 258-269.

[24] Mounaim, F., Sawan, M. and Bedard, S., Implantable Neuro-Monito-Stimulation System Dedicated to Enhance the Bladder Functions, IEEE Biomedical Circuits and Systems Conference - Healthcare Technology, London, UK, 2006, 198-201.

[25] Nadeau, P. and Sawan, M., A flexible high voltage biphasic current-controlled stimulator, Conference on Biomedical Circuits and Systems, 2006, 206-209.

[26] Stein, A. G., Eder, H. G., Blum, D. E., Drachev, A. and Fisher, R. S., An automated drug delivery system for focal epilepsy, Epilepsy research, 39, 2000, 103-114.

[27] David, J. A., Ortega, E. and Robert S. F., Diazepam prophylaxis for bicuculline-induced seizures: a rat dose-response model, Neuroscience Letters, 356, 2004, 66-68.

[28] Fisher, R. S. and Ho J., Potential new methods for antiepileptic drug delivery, CNS drugs, 16(9), 2002, 579-593.

[29] Oommena, J., Krausb, A. C., and Fisher, R. S., Intraventricular administration of gabapentin in the rat increases flurothyl seizure threshold, Neuroscience Letters, 417, 2007, 308-311.

[30] Anschel, D. J., Ortega, E. L., Kraus, A. C. and Fisher, R. S., Focally injected adenosine prevents seizures in the rat, Experimental Neurology, 190, 2004, 544-547.

[31] Katz, B. and Miledi, R., The effect of temperature on the synaptic delay at the neuromuscular junction, J. Physiol, 181, 1965, 656-670.

[32] Thompson, S. M., Masukawa, L. M. and Prince, D. A., Temperature dependence of intrinsic membrane properties and synaptic potentials in hippocampal CA1 neurons in vitro, J Neurosci, 5 1985, 817-824.

[33] Volgushev, M., Vidyasagar, T. R., Chistiakova, M., Yousef, T. and Eysel, U. T., Membrane properties and spike generation in rat visual cortical cells during reversible cooling, $J$ Physiol (London), 522(Pt. 1), 2000, 59-76.

[34] Xiao-Feng, Y., Duffy, D. W., Morley, R. E., and Rothman, S. M., Neocortical seizure termination by focal cooling: Temperature dependence and automated seizure detection, Epilepsia, 43, 2002, 240-245.

[35] Tanaka, N., Fujii, M., Imoto, H., Uchiyama, J., Nakano, K., Nomura, S., Fujisawa, H., Kunitsugu, I., Saito, T. and Suzuki, M., Effective suppression of hippocampal seizures in rats by direct hippocampal cooling with a Peltier chip, J Neurosurg., 108(4), 2008, 791-797.

[36] Rothman, S. M., Smyth, M. D., Yang, X. F. and Peterson, G. P., Focal cooling for epilepsy: an alternative therapy that might actually work, Epilepsy Behav, 7, 2005, 214-221.

[37] Rychlicki, F., Zamponi, N., Trignani, R., Ricciuti, R. A., Iacoangeli, M. and Scerrati M., Vagus nerve stimulation: clinical experience in drug-resistant pediatric epileptic patients, Seizure, 15(7), 2006, $483-490$.

[38] Sethi, N., Labar, D., Ponticello, L., Torgovnick, J., Sethi, P. and Arsura, E., Treatment of Medically Refractory Epilepsy: A Review Of Vagus Nerve Stimulator, The Internet Journal of Neurology, 9(1), 2008. 
[39] Morrell, M., Brain stimulation for epilepsy: can scheduled or responsive neurostimulation stop seizures?, Neurology, 2006, 164-168.

[40] Boon P, Raedt R, de Herdt V, Wyckhuys T, Vonck K., Electrical stimulation for the treatment of epilepsy, Neurotherapeutics, 2009, 6(2):218-27.

[41] Gosselin, B., Robert, P. Y. and Sawan, M., A Scalable Design for Signal Conditioning and Digitization in Implantable Multi-Channel Neural Sensors, Computer Architecture for Machine Perception and Sensing, International Workshop, 2007, 73-74.

[42] Gosselin, B. and Sawan, M., A low-power integrated neural interface with digital spike detection and isolation, 14th IEEE International Conference on Electronics, Circuits and Systems, Marrakech, Morocco, 2007.

[43] Gosselin, B. and Sawan, M., An ultra low-power CMOS action potential detector, IEEE International Symposium on Circuits and Systems, Seattle, WA, 2008.

[44] Gosselin, B., Simard, V., Roy, J. F., Marrouche, W., Dumortier, C. and Sawan, M., Multichannel wireless cortical recording: Circuits, system design and assembly challenges, IEEE International Workshop on Biomedical Circuits and Systems, Singapore, 2004.

[45] Gosselin, B., Simard, V. and Sawan, M., Low-power implantable microsystem intended to multichannel cortical recording, IEEE International Symposium on Circuits and Systems, Vancouver, BC, Canada, 2004.

[46] Gosselin, B., Simard, V. and Sawan, M., An ultra low-power chopper stabilized front-end for multichannel cortical signals recording, IEEE CCECE, 2004, 2259-2262. 



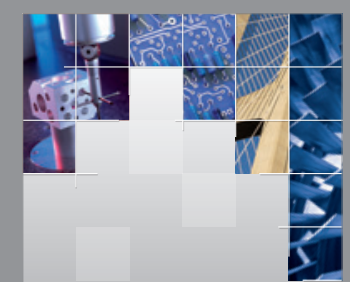

\section{Enfincering}
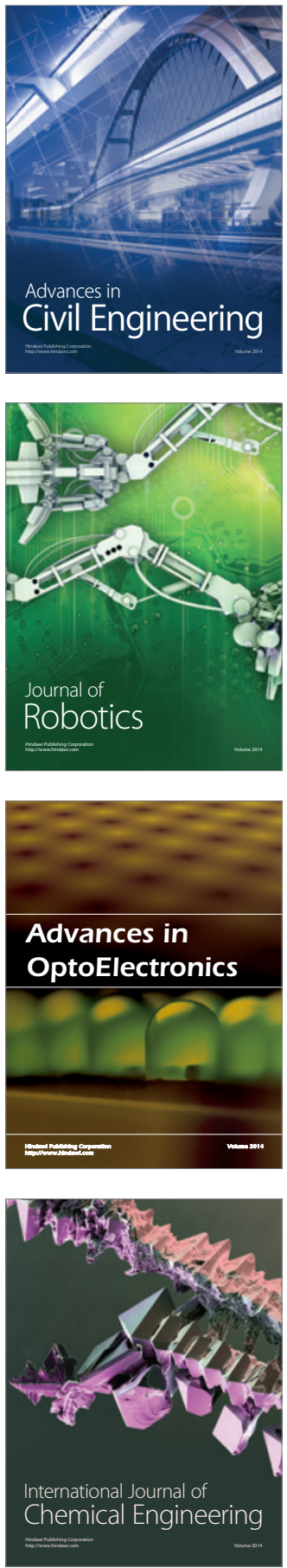

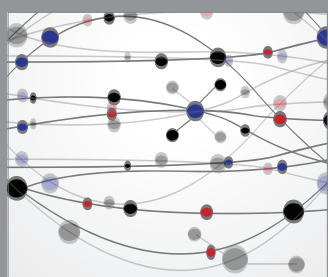

The Scientific World Journal

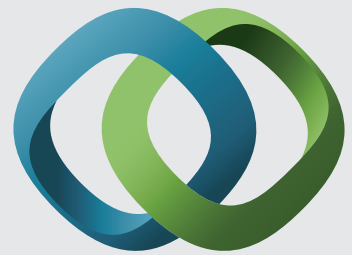

\section{Hindawi}

Submit your manuscripts at

http://www.hindawi.com
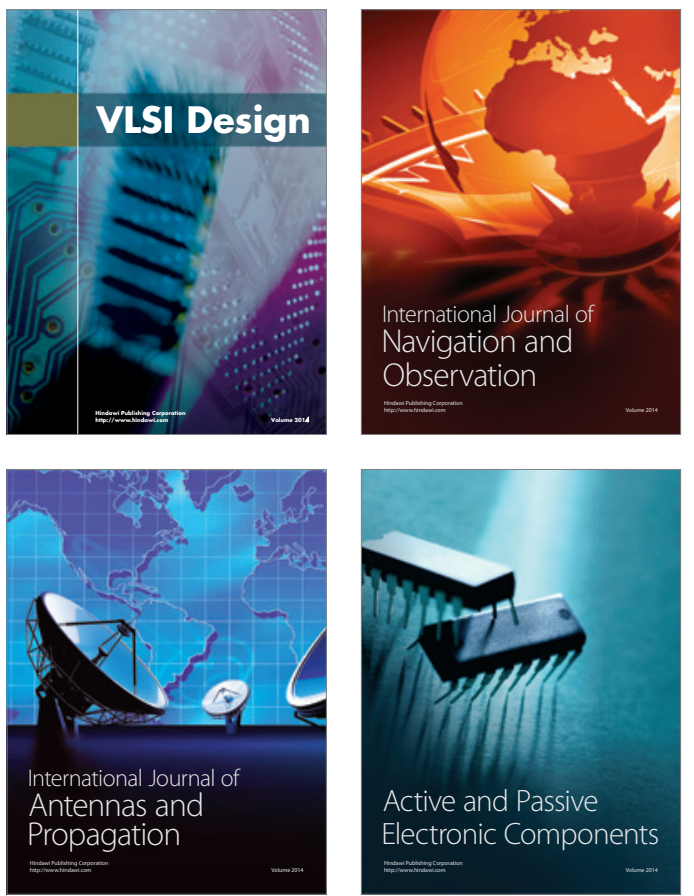
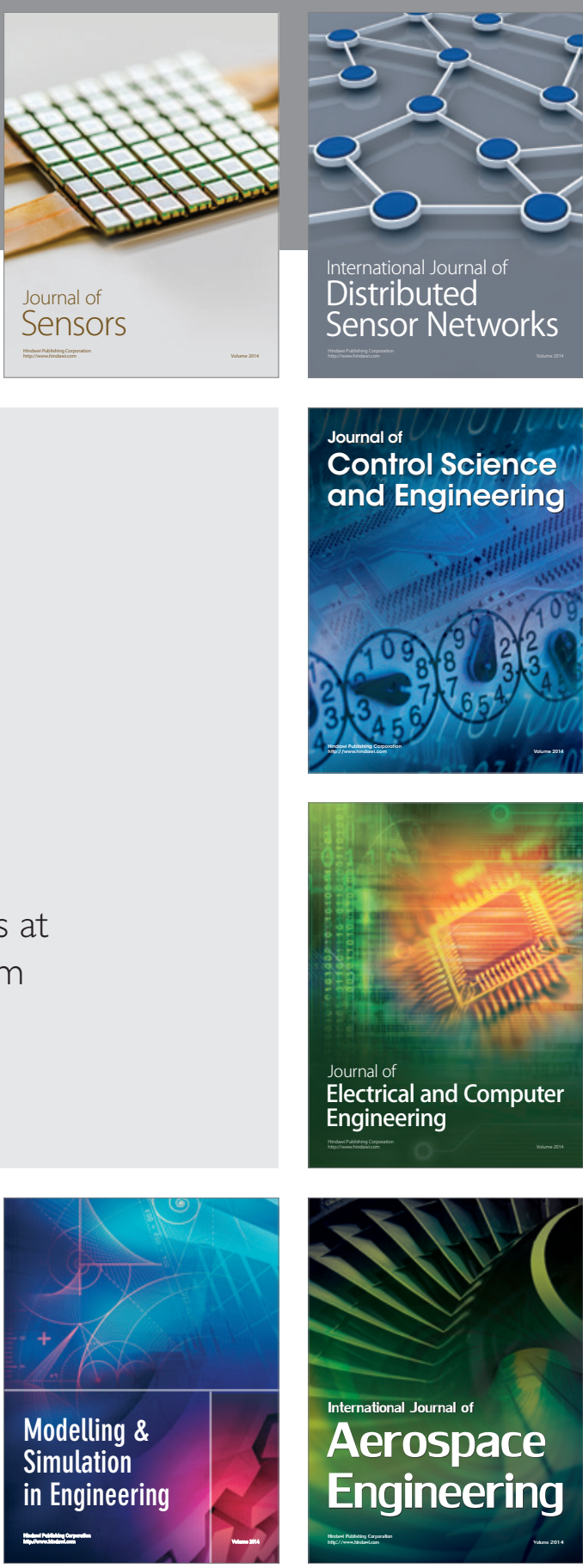

International Journal of

Distributed

Sensor Networks

Journal of

Control Science

and Engineering
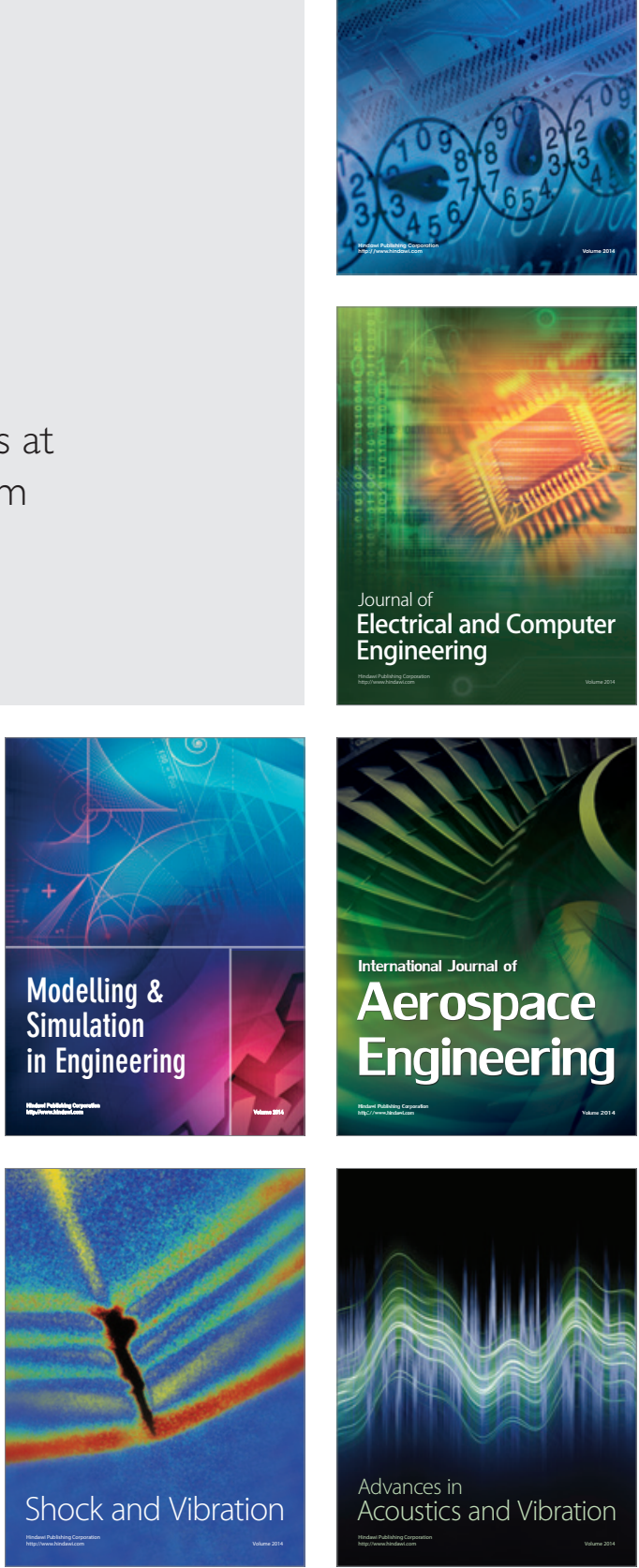